\section{Novel SARS-like Betacoronaviruses in Bats, China, 2011}

\section{Li Yang, ${ }^{1}$ Zhiqiang Wu, ${ }^{1}$ Xianwen Ren, ${ }^{1}$ Fan Yang, ${ }^{1}$ Guimei He, Junpeng Zhang, Jie Dong, Lilian Sun, Yafang Zhu, Jiang Du, Shuyi Zhang, and Qi Jin}

To clarify the evolutionary relationships among betavoronaviruses that infect bats, we analyzed samples collected during 2010-2011 from 14 insectivorous bat species in China. We identified complete genomes of 2 novel betacoronaviruses in Rhinolophus pusillus and Chaerephon plicata bats, which showed close genetic relationships with severe acute respiratory syndrome coronaviruses.

$\mathrm{T}$ he 2003 outbreak of severe acute respiratory syndrome (SARS) was caused by a novel betacoronavirus and rapidly spread globally, causing $\approx 8,000$ cases and nearly 900 deaths $(1,2)$. In June 2012, a novel betacoronavirus (called human coronavirus EMC [HCoV-EMC]) also was isolated from the sputum of a patient from Saudi Arabia who died of pneumonia and renal failure (3). Similar viruses were detected in 2 additional patients who had severe pneumonia in Qatar in September 2012 and in Saudi Arabia in November $2012(4,5)$. The clinical picture was remarkably similar to that of SARS and illustrates the epidemic potential of a novel coronavirus $(\mathrm{CoV})$ to threaten global health. SARS-CoVs and HCoV-EMC were suspected of spreading from bats to humans because these $\mathrm{CoVs}$ were most closely related to bat CoVs $(1,4)$. To clarify the evolutionary relationships among betavoronaviruses that infect bats, we analyzed samples collected during 2010-2011 from 14 insectivorous bat species common in 8 provinces in China.

\section{The Study}

We obtained pharyngeal and anal swab specimens of 414 insectivorous bats. Samples of each species were pooled and then processed with a viral particle-protected

Author affiliations: Ministry of Health Key Laboratory of Systems Biology of Pathogens, Beijing, China (L. Yang, Z. Wu, X. Ren, F. Yang, J. Dong, L. Sun, Y. Zhu, J. Du, Q. Jin); Institute of Pathogen Biology, Beijing (L. Yang, Z. Wu, X. Ren, F. Yang, J. Dong, L. Sun, Y. Zhu, J. Du, Q. Jin); and East China Normal University, Shanghai, China (G. He, J. Zhang, S. Zhang)

DOI: http://dx.doi.org/10.3201/eid1906.121648 nucleic acid purification method (6). The extracted RNA and DNA were amplified by sequence-independent PCR. The amplified viral nucleic acid libraries of the bat species were then sequenced with the Illumina/Solexa GAII sequencer (Illumina, San Diego, CA, USA). Those reads generated by the Illumina/Solexa GAII with length of 80 bases were directly aligned to the protein sequences in the National Center for Biotechnology Information nonredundant protein database by the blastx program in the BLAST software package, version 2.2.22 (www.ncbi. nlm.nih.gov/blast) with parameters "-e 1e-5 -F T -b 10 -v 10." No assembly was performed before alignment. Sequence similarity-based taxonomic assignments were conducted as described (7). We found 1,075 reads of betacoronavirus in Rhinolophus pusillus bats in Shaanxi and 92 reads of betacoronavirus in Chaerephon plicata bats in Yunnan.

We estimated the approximate locations of those reads on the $\mathrm{CoV}$ genome and their relative distances on the basis of alignment results exported with MEGAN 4MetaGenome Analyzer (http://ab.inf.uni-tuebingen.de/ software/megan/). The located reads were then used for reads-based nested PCR to identify genomic sequences. We established the complete genome sequences of 2 betacoronaviruses (Bat Rp-coronavirus/Shaanxi2011 and Bat Cp-coronavirus/Yunnan2011), which are 29,484 nt and 29,452 nt, respectively. The G+C content of Bat Rp-coronavirus/Shaanxi2011 and Bat Cp-coronavirus/ Yunnan 2011 is $41.6 \%$ and $40.9 \%$, respectively.

We conducted complete genome comparison and phylogenetic analysis on the basis of polymerase and spike protein. Pairwise genome sequence alignment was conducted by using EMBOSS Needle software (www.ebi.ac.uk/Tools/psa/emboss_needle/) with default parameters. The overall nucleotide sequences between Bat Rp-coronavirus/Shaanxi2011 and Bat Cp-coronavirus/ Yunnan 2011 indicated $88.7 \%$ nt identity. They shared $87.4 \%-89.5 \%$ nt identity with SARS-CoV, $88 \%-89.9 \%$ nt identity with the bat SARS-like CoV (bat SARSCoV Rm1), and $87.6 \%-89.6 \%$ nt identity with the civet SARS-like CoV (civet SARS-CoV SZ16). On the other hand, comparison between the betacoronavirus genomes and human betacoronavirus (HCoV-OC43) showed only $49.9 \%-50.4 \%$ nt overall identity, whereas the betacoronavirus genomes and HCoV-EMC showed 52.1\% nt overall identity.

The RNA-dependent RNA polymerase (RdRp, the 12th nonstructural protein codified to open reading frame $1 \mathrm{a}, \mathrm{b})$ is a highly conserved gene of CoVs, which is frequently used for phylogenetic comparison $(8,9)$. MEGA5.0 (www.megasoftware.net) was used to construct

${ }^{1}$ These authors contributed equally to this article. 
the phylogenetic trees on the basis of the nucleotide sequences and deduced amino acid sequences. First, we used the MUSCLE package and default parameters (www. megasoftware.net/) to construct the alignment. The best substitution model was then evaluated with the Model Selection package implemented in MEGA5. Finally, we used the maximum-likelihood method with an appropriate model to process the phylogenetic analysis with 1,000 bootstrap replicates. We constructed a phylogenetic tree based on the nucleotide sequences of the $R d R p$ gene to show the evolutionary relationship between these 2 betacoronaviruses and other CoVs (Figure 1). Reference $\mathrm{CoV}$ genome sequences were downloaded from GenBank and aligned with the fragments of the newly discovered CoVs. The RdRp genes of Bat Rp-coronavirus/

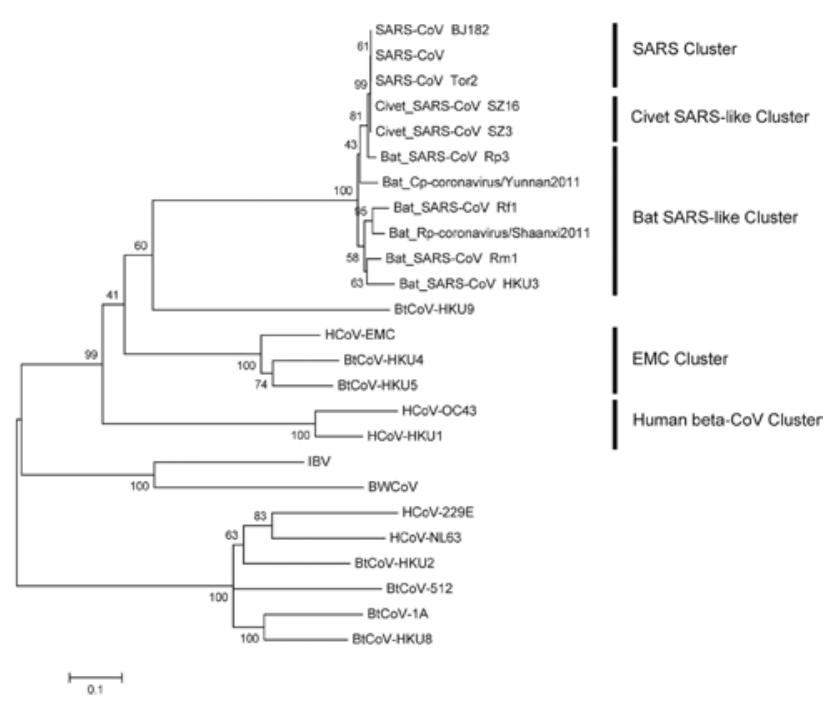

Figure 1. Phylogenetic tree of novel betacoronaviruses based on the nucleotide sequence of the RdRp gene. The following coronaviruses (CoVs) and GenBank accession numbers were used: bat severe acute respiratory syndrome CoV Rm1 (bat SARS-CoV Rm1; DQ412043), bat SARS-CoV Rp3 (DQ071615), bat SARSCoV Rf1 (DQ412042), bat SARS-CoV HKU3 (DQ022305),SARSCoV isolate Tor2/FP1-10895 (SARS-CoV Tor2; JX163925), SARSCoV BJ182-12 (SARS-CoV BJ182; EU371564), SARS-CoV (NC004718), civet SARS-CoV SZ3 (AY304486), civet SARS-CoV SZ16 (AY304488), bat CoV HKU9 (BtCoV-HKU9; EF065513), bat CoV HKU4 (BtCoV-HKU4; EF065505), bat CoV HKU5 (BtCoVHKU5; EF065509), human betacoronvirus 2c EMC/2012 (HCoVEMC; JX869059), human CoV OC43 (HCoV-OC43; NC005147), HCoV-HKU1 (NC006577), bat coronavirus HKU2 (BtCoV-HKU2; NC009988), bat coronavirus 1A (BtCoV-1A; NC010437), HCoV229E (NC002645), HCoV-NL63 (NC005831), bat CoV HKU8 (BtCoV-HKU8; NC010438), scotophilus bat CoV 512 (BtCoV-512; NC009657), avian infectious bronchitis virus (IBV; NC001451), beluga whale CoV SW1 (BWCoV; NC010646). Scale bar indicates genetic distance estimated by using $\mathrm{TN} 93+\mathrm{G}+\mathrm{I}$ model implemented in MEGA5 (www.megasoftware.net).
Shaanxi2011 and Bat Cp-coronavirus/Yunnan2011 were highly similar, sharing $93.1 \% \mathrm{nt}$ identity. The phylogenetic analysis demonstrated that betacoronaviruses and the bat SARS-like CoVs in our study are clustered (93.1\%-93.4\% nt identity) and are close in distance to SARS-CoVs (92.9\%-94.8\% nt identity) and civet SARS-like CoVs (93.1\%-94.8\% nt identity) but that bat $\mathrm{CoV}$ (BtCoVHKU9) and HCoV-OC43 are placed among the relatively distant groups $(65.8 \%-65.9 \%$ and $62.9 \%-63.5 \% \mathrm{nt}$ identities with the betacoronaviruses, respectively). Therefore, collectively we called these betacoronaviruses and bat SARS-like CoVs the bat SARS-like cluster of CoVs. Bat Rp-coronavirus/Shaanxi2011 and Bat Cpcoronavirus/Yunnan2011 showed little genetic similarity $(<66.2 \%-67.3 \%$ nt identity) to HCoV-EMC.

The spike proteins of $\mathrm{CoVs}$ are responsible for receptor binding and host species adaptation, and their genes therefore constitute one of the most variable regions within CoV genomes $(10,11)$. The phylogenetic tree based on the amino acid sequences of spike protein (Figure 2) suggests that the selected betacoronaviruses were mainly divided into 5 clusters: SARS cluster; bat SARS-like cluster; civet SARS-like cluster; human betacoronavirus cluster; and EMC cluster. Bat Rp-coronavirus/Shaanxi2011 and Bat Cp-coronavirus/Yunnan2011 shared $89.4 \%$ aa identity in spike proteins, which consisted of 1,240 aa and 1,241 aa, respectively. The spike proteins of the CoVs in our analysis have $89.8 \%-92.7 \%$ aa identity with those of bat SARS-like CoVs, with substantial similarity in the receptor-binding domain. The close relationship also was observed with the SARS-CoVs (79.2\%-79.4\% aa identity) and civet SARS-like CoVs (78.9\%-79.1\% aa identity). In contrast, the human betacoronaviruses and EMC cluster formed separate clusters distinct from SARS-related CoVs that showed only $27.8 \%-29.4 \%$ aa and $28.8 \%-30.5 \%$ aa identities with the betacoronaviruses, respectively, in our analysis. The genome sequences reported here have been deposited into GenBank (accession nos. JX993987-JX993988).

\section{Conclusions}

The recent fatal human infection caused by $\mathrm{HCoV}$ EMC has boosted interest in the discovery of novel CoVs in humans and animals. HCoV-EMC is a novel betacoronavirus, and its closest known relatives are BtCoVs HKU4, and HKU5, which have been detected in Hong Kong only in bats (12), the same animal from which SARS is believed to have originated. Bats are increasingly recognized as natural reservoirs of $\mathrm{CoVs}$ and may serve as intermediate hosts for interspecies transmission of SARSCoVs $(10,13)$. Different bat populations from various countries harbor diverse CoVs that have a high frequency of recombination and mutation rates that enable them to 


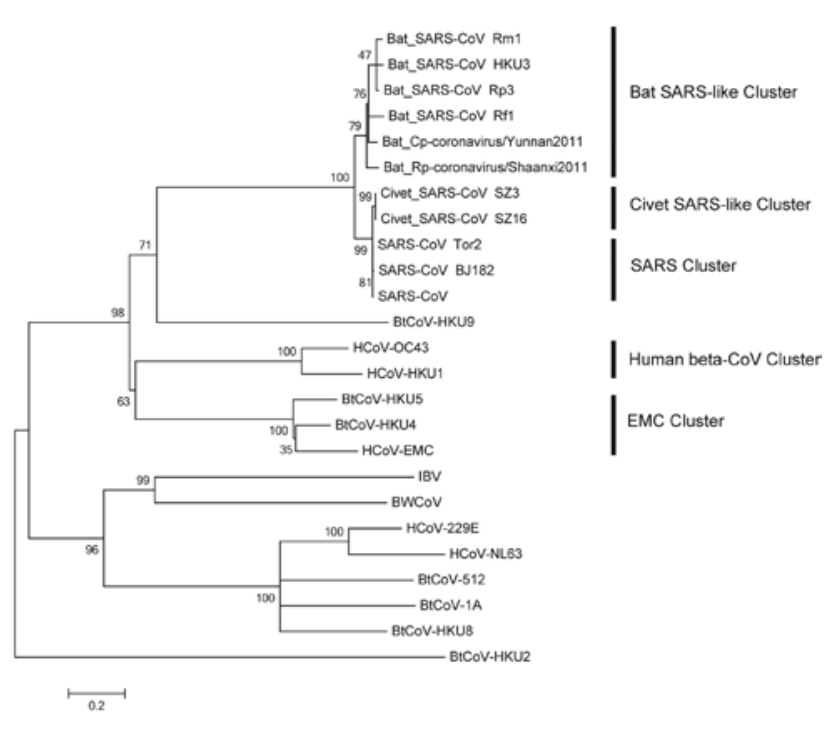

Figure 2. Phylogenetic tree of novel betacoronaviruses based on the deduced amino acid sequence of spike protein. SARS, severe acute respiratory syndrome; CoV, coronavirus; $\mathrm{HCoV}$, human CoV; BtCoV, bat CoV; BWCoV, beluga whale CoV; IBV, avian infectious bronchitis. Scale bar indicates genetic distance estimated by using $W A G+G+1+F$ model implemented in MEGA5 (www.megasoftware.net).

adapt to new hosts and ecologic niches $(14,15)$. Therefore, continuous studies of CoVs from different bat species and different countries would help better prevent the new global pandemics resulting from novel viral infection.

We detected and characterized 2 novel betacoronaviruses-Bat Rp-coronavirus/Shaanxi2011 in R. pusillus bats and Bat Cp-coronavirus/Yunnan2011 in C. plicata bats-in China. The high similarity shown by phylogenetic analysis confirmed the close genetic relationship among the CoVs (SARS-like CoVs and SARS-CoVs) that we analyzed. In contrast, Bat Rpcoronavirus/Shaanxi2011 and Bat Cp-coronavirus/ Yunnan2011 showed little genetic similarity with human betacoronaviruses and HCoV-EMC. Although several CoVs are found in horseshoe bats (Rhinolophus spp.), to our knowledge, the SARS-like CoVs in R. pusillus and C. plicata bats in China have not been identified. The description presented here will further the understanding of CoVs distribution in different bat species found in human habitats and provide clues for rapid response to potential public health threats.

This work was supported by the National Science and Technology Major Project, "China Mega-Project for Infectious Disease" (grant no. 2011ZX10004-001) from China.

Dr Li Yang is a research assistant at the Institute of Pathogen Biology in Beijing. Her research interest is virus discovery.

\section{References}

1. Peiris JS, Yuen KY, Osterhaus AD, Stohr K. The severe acute respiratory syndrome. N Engl J Med. 2003;349:2431-41. http:// dx.doi.org/10.1056/NEJMra032498

2. Drosten C, Gunther S, Preiser W, van der Werf S, Brodt HR, Becker $\mathrm{S}$, et al. Identification of a novel coronavirus in patients with severe acute respiratory syndrome. N Engl J Med. 2003;348:1967-76. http://dx.doi.org/10.1056/NEJMoa030747

3. Zaki AM, van Boheemen S, Bestebroer TM, Osterhaus AD, Fouchier RA. Isolation of a novel coronavirus from a man with pneumonia in Saudi Arabia. N Engl J Med. 2012;367:1814-20. http://dx.doi. org/10.1056/NEJMoa1211721

4. Corman V, Eckerle I, Bleicker T, Zaki A, Landt O, EschbachBludau M, et al. Detection of a novel human coronavirus by realtime reverse-transcription polymerase chain reaction. Euro Surveill. 2012;17: pii: 20285.

5. ProMEDmail. Novel coronavirus-Saudi Arabia: new case. ProMED-mail 2012 Nov 04 [cited 2012 Nov 4]. http://www. promedmail.org/, article no. 20121104.1391285.

6. Wu Z, Ren X, Yang L, Hu Y, Yang J, He G, et al. Virome analysis for identification of novel mammalian viruses in bat species from Chinese provinces. J Virol. 2012;86:10999-1012. http://dx.doi. org/10.1128/JVI.01394-12

7. Yang J, Yang F, Ren L, Xiong Z, Wu Z, Dong J, et al. Unbiased parallel detection of viral pathogens in clinical samples by use of a metagenomic approach. J Clin Microbiol. 2011;49:3463-9. http:// dx.doi.org/10.1128/JCM.00273-11

8. Woo PC, Lau SK, Chu CM, Chan KH, Tsoi HW, Huang Y, et al. Characterization and complete genome sequence of a novel coronavirus, coronavirus HKU1, from patients with pneumonia. J Virol. 2005;79:884-95. http://dx.doi.org/10.1128/JVI.79.2.884-895.2005

9. Lau SK, Li KS, Tsang AK, Shek CT, Wang M, Choi GK, et al. Recent transmission of a novel alphacoronavirus, bat coronavirus HKU10, from Leschenault's rousettes to Pomona leaf-nosed bats: first evidence of interspecies transmission of coronavirus between bats of different suborders. J Virol. 2012;86:11906-18. http://dx.doi. org/10.1128/JVI.01305-12

10. Li W, Shi Z, Yu M, Ren W, Smith C, Epstein JH, et al. Bats are natural reservoirs of SARS-like coronaviruses. Science. 2005;310:676-9. http://dx.doi.org/10.1126/science.1118391

11. Lau SK, Woo PC, Li KS, Huang Y, Wang M, Lam CS, et al. Complete genome sequence of bat coronavirus HKU2 from Chinese horseshoe bats revealed a much smaller spike gene with a different evolutionary lineage from the rest of the genome. Virology. 2007;367:428-39. $\mathrm{http}: / / \mathrm{dx}$.doi.org/10.1016/j.virol.2007.06.009

12. Woo PC, Lau SK, Li KS, Poon RW, Wong BH, Tsoi HW, et al. Molecular diversity of coronaviruses in bats. Virology. 2006;351:180-7. http://dx.doi.org/10.1016/j.virol.2006.02.041

13. Balboni A, Battilani M, Prosperi S. The SARS-like coronaviruses: the role of bats and evolutionary relationships with SARS coronavirus. New Microbiol. 2012;35:1-16.

14. Lau SK, Lee P, Tsang AK, Yip CC, Tse H, Lee RA, et al. Molecular epidemiology of human coronavirus OC43 reveals evolution of different genotypes over time and recent emergence of a novel genotype due to natural recombination. J Virol. 2011;85:11325-37. http://dx.doi.org/10.1128/JVI.05512-11

15. Woo PC, Lau SK, Huang Y, Yuen KY. Coronavirus diversity, phylogeny and interspecies jumping. Exp Biol Med (Maywood). 2009;234:1117-27. http://dx.doi.org/10.3181/0903-MR-94

$\overline{\text { Address for correspondence: Qi Jin, Ministry of Health Key Laboratory }}$ of Systems Biology of Pathogens, Institute of Pathogen Biology, Chinese Academy of Medical Sciences and Peking Union Medical College, No. 6, Rongjing East St, BDA, Beijing 100176, China; email: zdsys@vip.sina.com 